\title{
Chemical Surface Modification and Characterization of Carbon Nanostructures Without Shape Damage
}

\author{
Maria Clara Guimarães Pedrosa ${ }^{a}$, José Carlos Dutra Filho ${ }^{a}$, Livia Rodrigues de Menezes ${ }^{a}$ (D), \\ Emerson Oliveira da Silva * (1) \\ ${ }^{a}$ Universidade Federal do Rio de Janeiro, Centro de Tecnologia, Instituto de Macromoléculas \\ Professora Eloisa Mano, Rio de Janeiro, RJ, Brasil.
}

Received: August 30, 2019; Revised: February 10, 2020; Accepted: March 26, 2020

\begin{abstract}
Carbon nanostructures have been extensively studied due to combination of properties they present. With different geometries and reactivities, carbon nanotubes, graphene and fullerene were oxidized and modified with octadecylamine (ODA) in order to decrease van der Waals's interaction and consequently improve the polymer-carbon nanostructures affinity for use in nanocomposites. Nanostructures were characterized as morphology, chemical structure, molecular conformation and thermal properties. All nanostructures showed results that confirm the modification with ODA, without geometry being altered. Modified nanostructures showed different percentages of ODA: graphene $86 \%$, carbon nanotubes $83 \%$ and fullerene $63 \%$. It was attributed to distinct reactivities, shapes and specific surface area available, due to different amounts of reactive terminals obtained before oxidation reactions. However, they showed a lower interfacial interaction, which is highly favorable for use in polymer nanocomposites.
\end{abstract}

Keywords: fullerene, carbon nanotubes, graphene, octadecylamine.

\section{Introduction}

Carbon is a remarkable element in its ability to combine itself and other chemical elements in different ways and thus form the basis of organic chemistry and life. Carbon nanostructures are made up of carbon only and have different geometries and dimensions. However, these structures have distinct reactivities, which can be justified due different tensions between its curvatures. Graphene shows $\mathrm{sp}^{2}$ hybridization, whereas carbon nanotube and fullerene have same type of hybridization, but only deformed because of their geometries ${ }^{1}$.

Tension in nonplanar conjugated molecules results from two main sources: pyramidalization of conjugated carbon atoms and misalignment of $\pi$-orbital between adjacent pairs of conjugated carbon atoms. Pyramidalization angle $\left(\theta_{p}\right)$ is between an $\sigma$-orbital and a $\pi$-orbital on the same carbon atom $^{1,2}$. Therefore, carbon nanostructures present different pyramidalization angles, which are directly related to the distinct reactivity for each nanostructure.

Chemical reactions that saturate carbon atoms are highly favored due to relief of tension. In this way, the reactivity is favored according to geometry of nanostructure, varying according to increase of pyramidalization angle and misalignment of $\pi$-orbitals. Carbon nanotubes have a lower influence of curvature, but they present misalignment of $\pi$-orbitals that also favor reactivity. Thus, modifications in case of fullerene are more susceptible, since it presents the greatest tension between carbon bonds, when compared to nanotubes and later to graphene $e^{1,3}$.

Carbon nanostructures are constantly functionalized in search of compatibility with other materials, for example

*e-mail: eos@ima.ufrj.br. with polymers to produce nanocomposites with polymer matrix $^{4-6}$. These types of nanostructures tend to agglomerate due to intermolecular interaction of van der Waals, which hinders their dispersion when incorporated into a polymeric matrix. Functionalization is an alternative to avoid the agglomeration of nanostructures and increase interaction with polymer, usually through functional groups inserted by superficial modification ${ }^{7}$. However, changes in the chemical structure of the carbon nanoparticles may alter its properties. If this occurs, the nanocomposite does not show the desired properties. So, it is important to understand how superficial modification takes place and if the alteration in its properties is due to this modification or its geometry.

Fullerene was discover in 1985 by Kroto et al. ${ }^{8}$, and is a highly unsaturated spheroidal molecule, which may be composed of distinct amounts and pairs of carbon. $\mathrm{C}_{60}$ and $\mathrm{C}_{70}$ fullerene are the most abundant and representative ${ }^{9}$, but there are many already discovered fullerenes and the large range of fullerenes can be represented by $\mathrm{C}_{\mathrm{n}}$, where $\mathrm{n}=30-190^{9} . \mathrm{C}_{60}$ presents a combination of optical and electrical properties ${ }^{10}$. Arriagada and co-works studied the types of interaction between graphene and fullerene complexes with and without surface modifications. They showed that fullerenes are physisorbed on graphene while the chemisorption is reached on Fe-doped graphene nanosheets (cycloadditions) ${ }^{11}$.

Carbon nanotubes (CNT) have the combination of low density, high surface area and high aspect ratio ${ }^{12-15}$. Carbon nanotubes are allotropes formed by long cylinders of covalent bonds between carbon atoms, those are entirely of $\mathrm{sp}^{2}$ character. Moreover, the end of these nanotubes may or 
may not be closed by hemi-fullerenes. There are two types of structure in terms of number of layers: single-wall carbon nanotube (SWCNT), which consists of a single "seamless" rolled sheet of graphene and multi-wall carbon nanotube (MWCNT), which are composed of graphene cylinders aligned coaxially around a central hollow core with a layer spacing of approximately $0.34-0.36 \mathrm{~nm}^{7,14-16}$.

Graphene is obtained by exfoliation of graphite, presenting a single layer of carbons bound by $\mathrm{sp}^{2}$ hybridization. Novoselov and collaborators isolated the first sheet of graphene in 2004 , with the aid of an adhesive tape, thus performing a mechanical exfoliation. This nanostructure presents a combination of electrical, thermal and mechanical properties ${ }^{17,18}$.

The isolation of graphene sheets can be performed by chemical and mechanical exfoliation. Chemical exfoliation by oxidation is the most common, this modification generates chemical bonds in graphene sheets, thus facilitating unpacking of sheets. However, this exfoliation generally promotes defects in the structure of graphene, which can be detrimental in some properties of graphene, such as electric.

The aim of this study is to modify surface of different carbon nanostructures (multi-walled carbon nanotubes, graphene and $\mathrm{C}_{60}$ fullerene) with octadecylamine and to characterize the modified nanostructures regarding morphology, chemical structure, molecular conformation and thermal properties.

\section{Experimental}

Fullerene $\mathrm{C}_{60} 98 \%$ was purchased from Sigma Aldrich; multi-walled carbon nanotubes from Laboratory of Nanomaterials - Physics Department - ICEX - UFMG and graphite HC 30 from Grafmax were modified in this work. Octadecylamine $99.0 \%$, nitric acid $65 \%$ PA, sulfuric acid $95-99 \%$ PA and phosphoric acid $85 \%$ were supplied from Sigma Aldrich and used as received. Chloroform, ethanol $99.5 \%$ and potassium permanganate PA from Merck S.A. were used as received.

Fullerene $\mathrm{C}_{60}$ were treated with sulfuric acid $\left(\mathrm{H}_{2} \mathrm{SO}_{4}\right)$ and nitric acid $\left(\mathrm{HNO}_{3}\right)$, as received. The system was refluxed, stirred and heated at $100^{\circ} \mathrm{C}$ for 24 hours. After, product was washed with water, filtrated until $\mathrm{pH}$ was equal to that of pure water, and dried for 48 hours at $50{ }^{\circ} \mathrm{C}$. This oxidation was adapted from literature ${ }^{19-21}$.

Oxidized fullerene and octadecylamine (1:10) were heated at $65{ }^{\circ} \mathrm{C}$ and stirred for $24 \mathrm{~h}$. This procedure were adapted $^{19,22}$, but presents a differential that is not use a solvent. After this, to the system was added ethanol $(50 \mathrm{~mL})$ and subjected on ultrasound bath for 30 minutes. The solution was vacuum filtered through a $0.22 \mu \mathrm{m}$ membrane, washed with ethanol to remove excess of octadecylamine, and dried at $50{ }^{\circ} \mathrm{C}$ for 48 hours. The same treatment was used to carbon nanotubes ${ }^{19,22}$.

Graphite $(0.75 \mathrm{~g})$ was treated with a solution of sulfuric acid $\left(\mathrm{H}_{2} \mathrm{SO}_{4}\right)$, phosphoric acid $\left(\mathrm{H}_{3} \mathrm{PO}_{4}\right)(90: 10 \mathrm{~mL})$ and potassium permanganate $\left(4.5 \mathrm{~g} \mathrm{KMnO}_{4}\right)$, that was subjected on ultrasound bath for 30 minutes. After that, the system was refluxed, stirred and heated at $80^{\circ} \mathrm{C}$ for 24 hours. At the end of reaction, hydrogen peroxide $\left(2 \mathrm{~mL} \mathrm{H}_{2} \mathrm{O}_{2}\right)$ and $200 \mathrm{~mL}$ of ice were put in the reaction, and final solution was vacuum filtered through a $0.22 \mu \mathrm{m}$ membrane and washed with water.
It was filtrated until the $\mathrm{pH}$ was equal to that of pure water and after these; the product was dried for 48 hours at $50{ }^{\circ} \mathrm{C}^{23,24}$.

Graphene oxide were treated with octadecylamine (1:10), heated at $130{ }^{\circ} \mathrm{C}$ and stirred for 48 hours. After this, to the system was added ethanol $(50 \mathrm{~mL})$ and subjected on ultrasound bath for 30 minutes. The solution was vacuum filtered through a $0.22 \mu \mathrm{m}$ membrane and washed with ethanol to remove excess of octadecylamine. At the end, modified graphene was dried at $50{ }^{\circ} \mathrm{C}$ for 48 hours.

\section{$2.1 X$-ray diffraction measurements (XRD)}

XRD was used to determine structural changes in carbon nanostructures caused by modification steps. Bragg's Law was applied in order to relate $2 \theta$ values of reflection peaks and interplanar spacing (d). XRD patterns were obtained by using a Rigaku Ultima IV x-ray diffractometer with $\mathrm{CuK} \alpha$ radiation source $(\lambda=0.1541 \mathrm{~nm})$ operated at $40 \mathrm{kV}, 30 \mathrm{~mA}$, $0.05^{\circ} \mathrm{s}^{-1}$ at room temperature and interval degree $2 \theta=2$ to $50^{\circ}$.

\subsection{Fourier transform infrared spectroscopy measurements (FTIR)}

FTIR analysis were performed in range $4000-400 \mathrm{~cm}^{-1}$, analyzed at $25^{\circ} \mathrm{C}$ in a Perkin Elmer Spectrum release device:10.4.2 and Frontier model FT-IR/FIR zinc selenite crystal using $\mathrm{KBr}$ to analyses nanostructures.

\subsection{Thermal Gravimetric Analysis (TGA)}

Measurements were obtained through of Thermogravimetric Analyzer TGA Q-500 (TA Instruments). Samples were heat from 40 to $700{ }^{\circ} \mathrm{C}$, at heating rate of $5^{\circ} \mathrm{C} / \mathrm{min}$ and under nitrogen atmosphere (flow rate $=100 \mathrm{~mL} / \mathrm{min}$ ).

\subsection{Scanning Electron Microscope analysis (SEM)}

SEM analysis were carried out to identify morphology of carbon nanostructures modified and unmodified. The samples in powder form were fixed on surface of metal stubs with the aid of a carbon ribbon. These stubs were taken to Sputter Coater equipment BAL-TEC, SDC 005 and covered with a layer of gold. Analyses of carbon nanostructures were held in scanning electron microscope FEI Quanta 400. For fullerene modified with octadecylamine and oxidized carbon nanotubes were used the scanning electron microscope HITACHI, model TM3000.

\section{Results and Discussion}

By X-ray diffraction, it was observed that Fullerene features eight narrow crystalline peaks centered at $2 \theta=10.5^{\circ} ; 17.3^{\circ}$; $20.4^{\circ} ; 21.3^{\circ} ; 27.0^{\circ} ; 27.7^{\circ} ; 30.5^{\circ} ; 32.4^{\circ}$. Oxidized fullerene exhibits the same eight peaks but all with reduced light intensity and smooth extension of their bases (Figure 1a). The same phenomenon was observed by Oh et al. ${ }^{25}$ which indicates a lower degree of organization after oxidation.

Fullerene modified with octadecylamine has only two broad-based peaks which are centered $2 \theta=3^{\circ}$ and $21.2^{\circ}$. This result shows that organization of the particles of fullerene before modification with octadecylamine had a regularity and uniformity, while with modifier in the structure this 

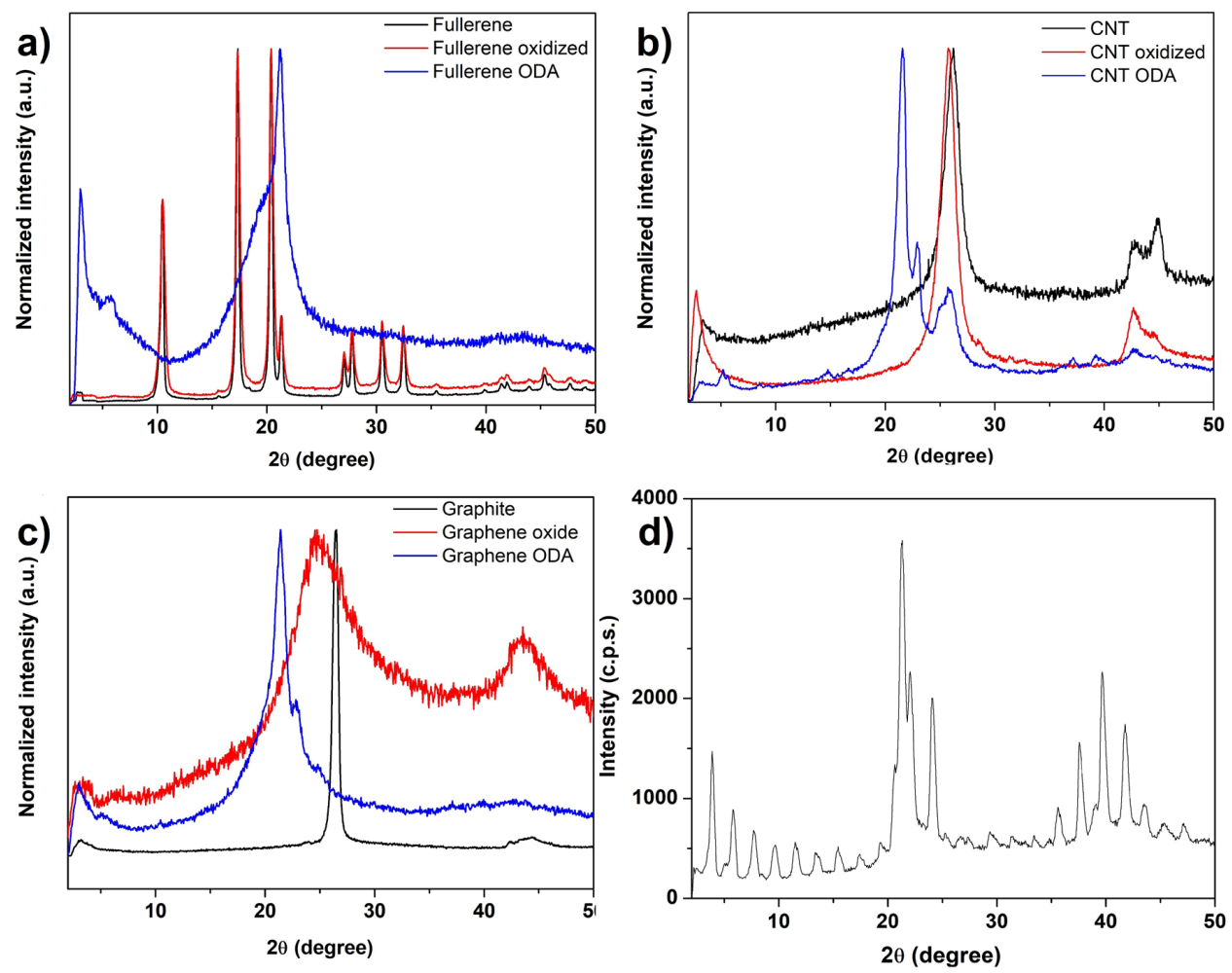

Figure 1. (a) XRD of fullerene, oxidized and modified with octadecylamine; (b) XRD of carbon nanotubes, oxidized and modified with octadecylamine; (c) XRD of graphite, graphene oxide and graphene ODA; (d) XRD of octadecylamine.

organization has been modified and has irregular and larger interplanar distances.

Through XRD, it is possible to calculate interplanar spacing and in the case of multiwall carbon nanotubes, the values can be found from distance between inner layers of nanotubes and the distance between carbon nanotubes, which tend to be agglomerated due to van der Waals interactions.

The peaks are found centered at $2 \theta$ equal to $26.2^{\circ}$; $42.8^{\circ}$ and $45^{\circ}$ (Figure 1b) with values for distance between nanotubes and between layers of $0.34 \mathrm{~nm} ; 0.21 \mathrm{~nm}$ and $0.20 \mathrm{~nm}$, respectively. According to Moniruzzaman and Winey (2006), the distance between walls for this type of carbon nanotube is about $0.34 \mathrm{~nm}$, even spacing between graphene sheets in graphite.

XRD results for oxidized carbon nanotube also exhibit a peak centered at $2 \theta$ equal to $25.8^{\circ}$ with a distance between walls of $0.34 \mathrm{~nm}$ nanotubes. The permanence of this distance shows that even with acid treatment for oxidation, the structural integrity of nanotubes was maintained. For carbon nanotubes modified with octadecylamine, peaks are centered at $21.6^{\circ} ; 22.9^{\circ}$ and $25.9^{\circ}$ with values for $\mathrm{d}$-spacing around $0.41 ; 0.39$ and $0.34 \mathrm{~nm}$, respectively (Figure 1b). The peak shift at $21.6^{\circ}$ shows that after modification with ODA nanotubes presented an organization with a greater distance between walls, but more organized than to oxidized nanotubes, which may be related to the interaction between ODA and nanotubes. The increase in distance between layers of nanotubes indicates that ODA chains have been inserted between walls of nanotubes causing distancing or opening of these layers.

Diffractogram obtained for graphite has a single peak crystalline, narrow and centered at $2 \theta=26.5^{\circ}$ (Figure 1c). Through this value was calculated interplanar spacing of graphene sheets using Bragg's Law, which is $0.34 \mathrm{~nm}$. The narrow peak characteristic indicates that graphite has a uniform distance between sheets ${ }^{21}$. After oxidation, were obtained graphene oxide, which presents two peaks with an extremely broad base, which denote a strong organizational change due to structural modification of carbon nanostructure ${ }^{26}$.

XRD for graphene oxide shows an increase of interplanar distances after oxidation, which is due to the presence of carboxyl groups and hydroxyls of graphene surfaces and broadening of peaks, indicates heterogeneity of distances between crystalline planes. When modified with octadecylamine, interplanar spacing was increased due to appearing of two peaks, because both are on lower $2 \theta$ values when compared with other structures. The first centered at $2 \theta=3{ }^{\circ}$ and second $2 \theta=21.4^{\circ}$. Through calculations by Bragg's Law, these peaks indicate interplanar spacing of $5.89 \mathrm{~nm}$ and $0.83 \mathrm{~nm}$, respectively.

The narrowing of peaks in sample Graphene ODA expresses minor variations for values of distances between planes, when compared with results for graphene oxide ${ }^{27}$. Therefore, indicating that in addition to increasing spacing, the modification method was also efficient in relation to periodicity with which interplanar distances were repeated. 
In order to understand the appearance of a peak at $21^{\circ}$ for all samples, an octadecylamine diffractogram was performed (Figure 1d) and this showed an intense peak centered at approximately $21^{\circ}$. Xu et al..$^{19}$ for carbon nanotubes modified with octadecylamine also report this phenomenon. According to authors, the most intense diffraction peak corresponds to crystallinity of octadecylamine.

FTIR analysis of modified nanostructures had as main intention to investigate the occurrence of chemical bonding between octadecylamine and the three carbon nanostructures evaluated. According to results obtained (Figure 2) was determined that modification occurred similarly for all nanostructured due to the appearance of bands related to bonding's vibrations $-\mathrm{CH}_{2}$ and $-\mathrm{CN}$, which are characteristic from octadecylamine.

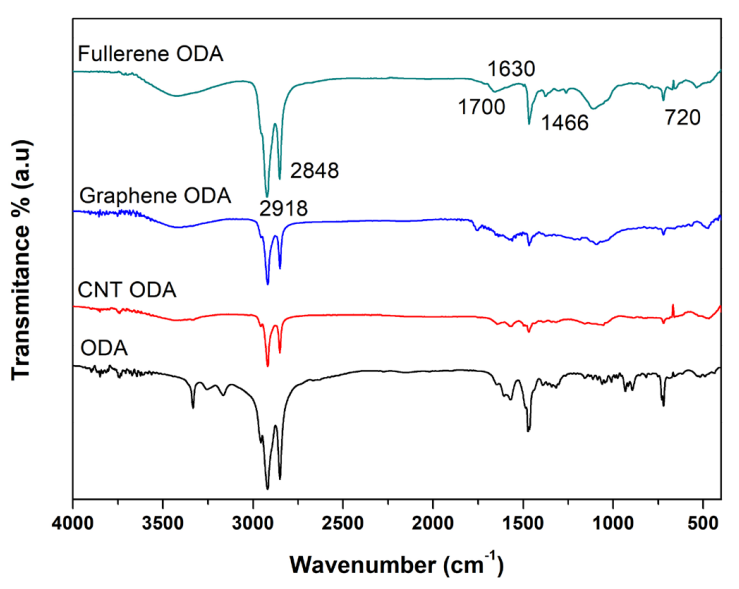

Figure 2. FTIR of octadecylamine and modified nanostructures.
Absorption bands at 2918 and $2848 \mathrm{~cm}^{-1}$ correspond to symmetric and asymmetric stretching vibrations and the rocking vibration at $720 \mathrm{~cm}^{-1}$ of aliphatic hydrocarbon groups $\mathrm{s}^{20,22,28-31}$. As well as the stretching vibration at $1466 \mathrm{~cm}^{-1}$ regarding $\mathrm{C}-\mathrm{N}$ amide bond between octadecylamine and the carbon nanostructures ${ }^{22,31,32}$.

FTIR spectrum also shows an axial deformation at 1700 to $1630 \mathrm{~cm}^{-1}$. The occurrence of this vibration demonstrates that octadecylamine is covalently bonded to each carbon nanostructures, since the reaction between carbonyl carbon (generated in the oxidation) and octadecylamine forms an amide function.

Another important point in the spectra of nanostructures is the absence of bands in range of 3400 to 3250 which corresponded to $\mathrm{NH}$ stretching and also of the range 1650 to 1580 of NH vibration bending, as both are characteristic of aliphatic primary amine (in all spectra of modified nanoparticles). Through this, it is possible to state that any unreacted amine has been successfully eliminated.

Thermal degradation curves of evaluated nanostructures allow us to observe its high thermal resistance in its modified state not denoted by reduced mass loss of these samples up to $700^{\circ} \mathrm{C}$ (Figure 3). Such behavior is already known in literature and is justified based on the high stability and structural rigidity of these particles ${ }^{30,33,34}$. After the oxidation process, it is possible to observe a slight reduction in thermal resistance thereof. This behavior reflects the structural defects created by the oxidation process, which reduces the stability of its structure and hence its rigidity, as previously observed by XRD.

The octadecylamine used in the modification of carbon nanostructures has a thermal resistance much lower than the
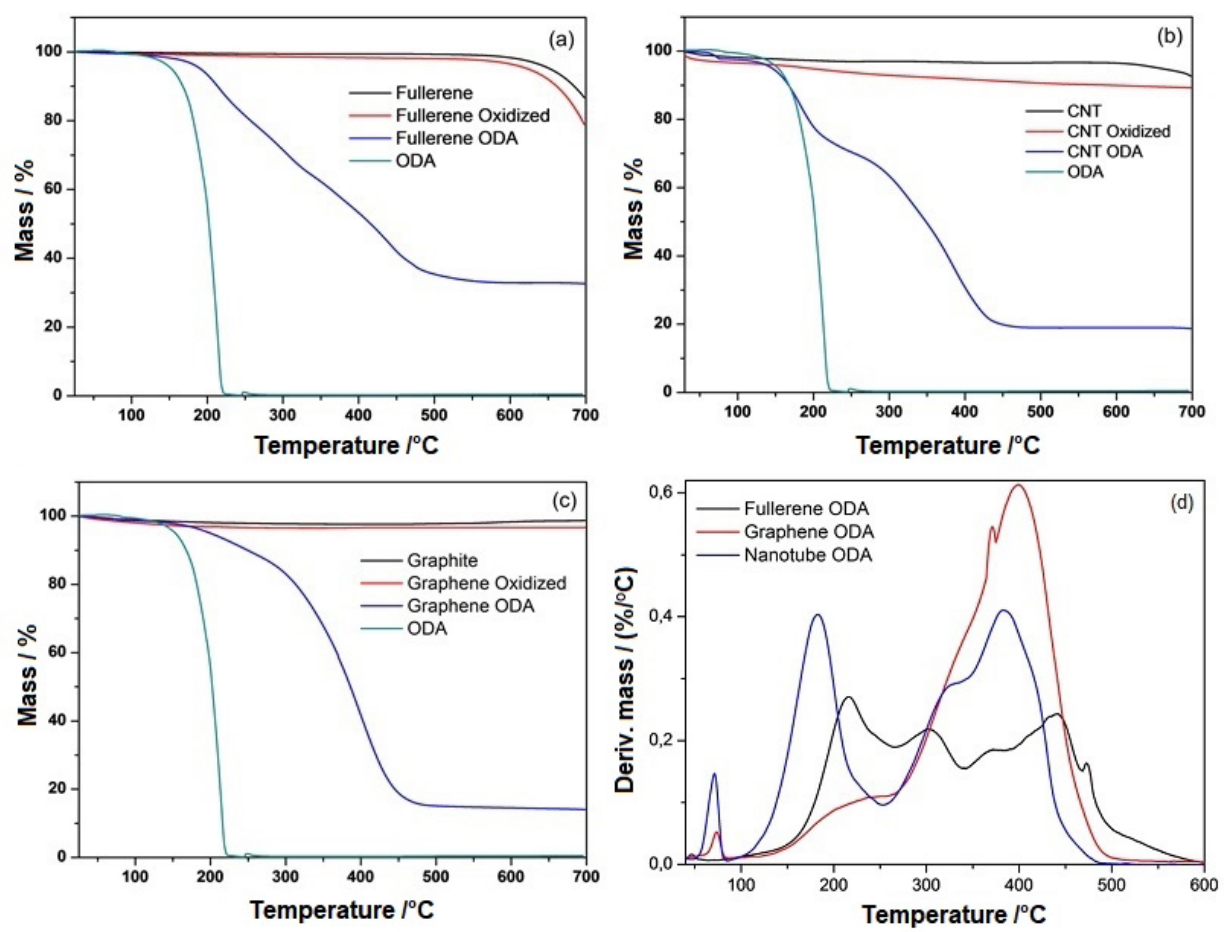

Figure 3. (a) TGA curves of octadecylamine and fullerenes; (b) TGA curves of octadecylamine and CNTs; (c) TGA curves of octadecylamine and modified graphenes; (d) DTG curves of nanostructures with ODA. 
particles and the degradation starts on temperatures below those observed for nanostructures $\left(\mathrm{T}_{\text {onset }}\right.$ approximately $\left.165^{\circ} \mathrm{C}\right)$. Thus, the incorporation of amine reduces the thermal resistance of nanostructures.

$\mathrm{T}_{\text {onset }}$ of modified nanostructures is concerning the start of the incorporated surface modifier degradation. In this perspective, although all particles exhibit a reduction of its heat resistance, it is remarkable that the beginning of the modifier loss is different for each geometry of evaluated particles.

According to previous studies, this behavior stems from the degree of thermal protection provided to amine by the greater or lesser intensity of interfacial interactions throughout their chain can establish with each of these nanostructures, giving the modifier chain a greater or lesser stiffness so influencing their resistance thermal ${ }^{35}$.

In the initial mass loss, one can observe a distinct profile of degradation curves of modified nanostructures. It was indicated by XRD that the octadecylamine chain was possibly inserted between nanotube layers. The thermal degradation profile of these particles corroborates this possibility, since are observed two instances well-defined mass loss, where both located at higher temperatures possibly correspond to degradation of octadecylamine chains with different thermal protection levels conferred by its position more superficial or more internal areas of carbon nanotubes.

TGA results of modified fullerene and graphene (Figure 3a and 3c, respectively) showed the existence of more than one loss event but in this case, is not possible to separate loss levels as observed for nanotube-ODA. The other data that can be extracted from thermograms are final residue and the slope on the main event mass loss. Although fullerene presents a $\mathrm{T}_{\text {onset }}$ at temperatures below those observed for graphene, the loss event is slower leading to an offset temperature higher than that observed for graphene.

Analysis of residues on modified nanostructures may indicate which one has a higher amount of amine on their surface. Given that, the carbon nanostructures in their oxidized state have little mass loss until $600^{\circ} \mathrm{C}^{36}$, one may define the losses observed until this temperature corresponding to inserted modifier its surface. Thus, the largest insertion amine was in graphene, followed by nanotube and fullerene ${ }^{30}$.

The difference in insertion of amine in each nanostructure occurs possibly because of their different geometries correlated with the extension of chain amine used in the modification process. Graphene and nanotubes showed very close mass loss of values among themselves, which included a loss of $86 \%$ and $83 \%$, respectively.

Fullerene lost just $63 \%$ indicating the presence of a minor amount of amine incorporated. In this case, it should be considered that its spherical shape formed by only 60 carbons when oxidized leads the formation of terminals really close which may be limited to the bonding of amine due to higher steric hindrance. While graphene and nanotube, due to the high aspect ratio, have these more distant terminals. Moreover, the difference in oxidation processes may have originated a greater number of reactive terminals on the surface of these nanostructures increasing its modification.

DTG curves denote the complex pattern of weight loss of the modified nanostructures (Figure $3 \mathrm{~d}$ ). In all these samples, it can notice an initial loss between 60 and $80^{\circ} \mathrm{C}$, although this loss can be observed in DTG available in the literature, there are no reports about their origin. However, after the modification of the nanostructures it is possible that these will have absorbed moisture after the drying. This is possible due to the presence of hydrophilic terminals, which may have absorbed moisture on the surface.

Images of SEM were captured in order to know geometry and morphology of carbon nanostructures before and after chemical surface modification. In Figure $4 \mathrm{a}$ and $4 \mathrm{~b}$ it is possible to note the decrease in size of the clusters with fullerene oxidation process, as well as increased surface roughness, thus evidencing oxidation. Image $4 \mathrm{c}$ shows

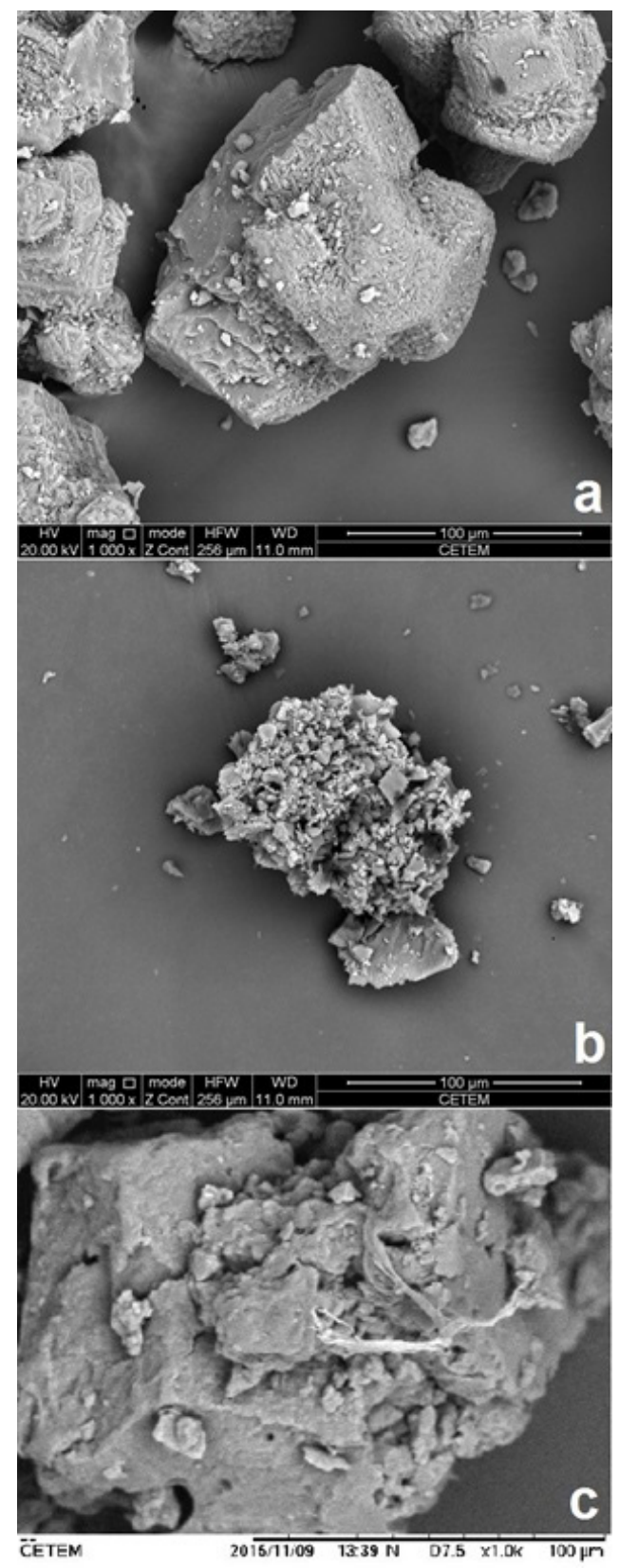

Figure 4. Images of SEM (a) fullerene $\mathrm{C}_{60}$; (b) oxidized fullerene and (c) fullerene modified with octadecylamine. 
change of surface with the chemical surface modification with octadecylamine as well as an increase of agglomerate, reinforcing the results revealed by FTIR, XRD and SEM.

Figure 5a shows multiwall carbon nanotubes as received. It is possible to see the high aspect ratio of the nanotubes. Figure $5 \mathrm{~b}$ shows nanotubes after oxidation and it is apparent that acid treatment did not affect the geometry of nanostructure, thus retaining its tubular form. However, it is observed that there was some structural disorganization, because there was the appearance of some clumps of tangled nanotubes, suggesting that there was surface oxidation ${ }^{37}$.

Figure $5 \mathrm{c}$ shows the modified carbon nanotubes with octadecylamine. In this image is still possible to view the morphology of tubes, but the presence of a mass, which is possibly the modifier between the nanotubes strongly entangled indicating that they actually suffered some superficial chemical modification, as previously observed by FTIR. The presence of multiwall on carbon nanotubes cannot be visualized by SEM, but were confirmed by distance found between nanotube walls in XRD analysis.

Figure 6 a shows graphite as received, which graphene sheets packaged with a distance between sheets that is not noticeable form. After the oxidation, graphene oxide was obtained in order to separate graphene sheets ${ }^{38}$. Figure $6 \mathrm{~b}$ shows graphene oxide on a level of disorganization of material and a greater distance between sheets that indicates there was an expansion of graphite due to the aspect crumpled and exfoliated ${ }^{39}$. Figure $6 \mathrm{c}$ shows graphene modified with
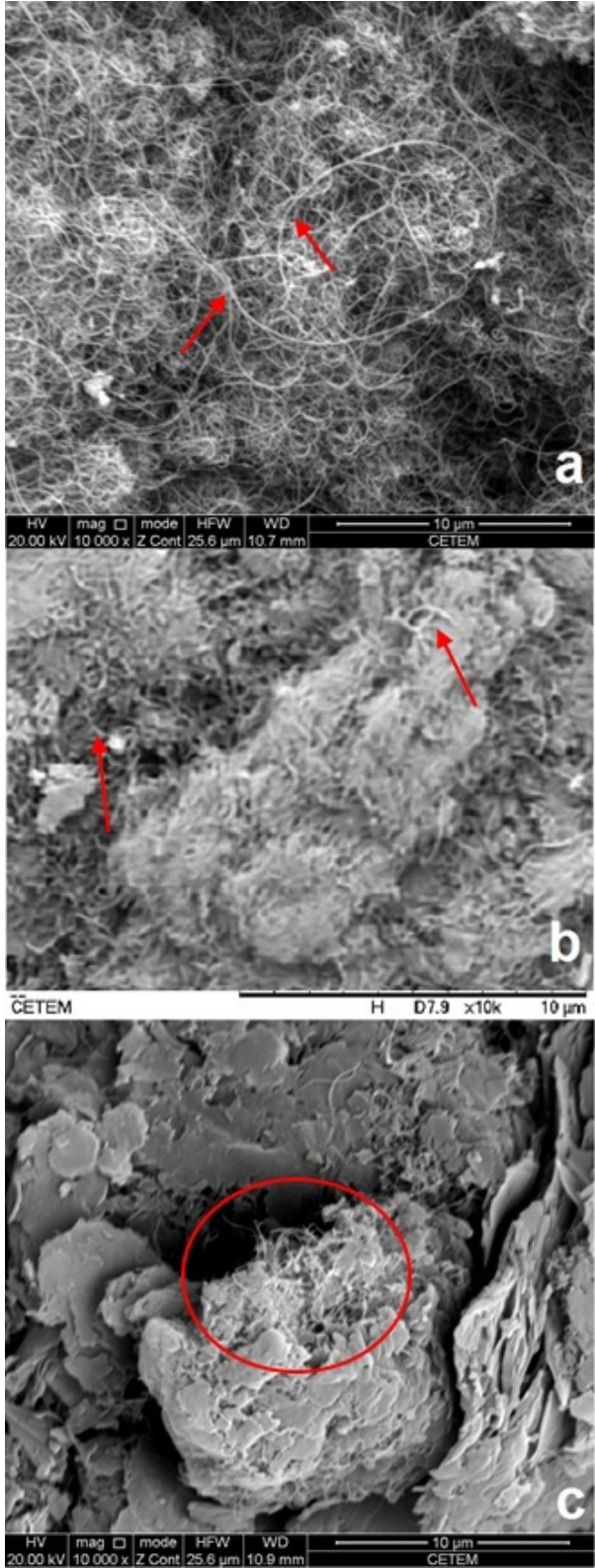

Figure 5. Images of SEM (a) CNT; (b) oxidized CNT and (c) CNT modified with octadecylamine.

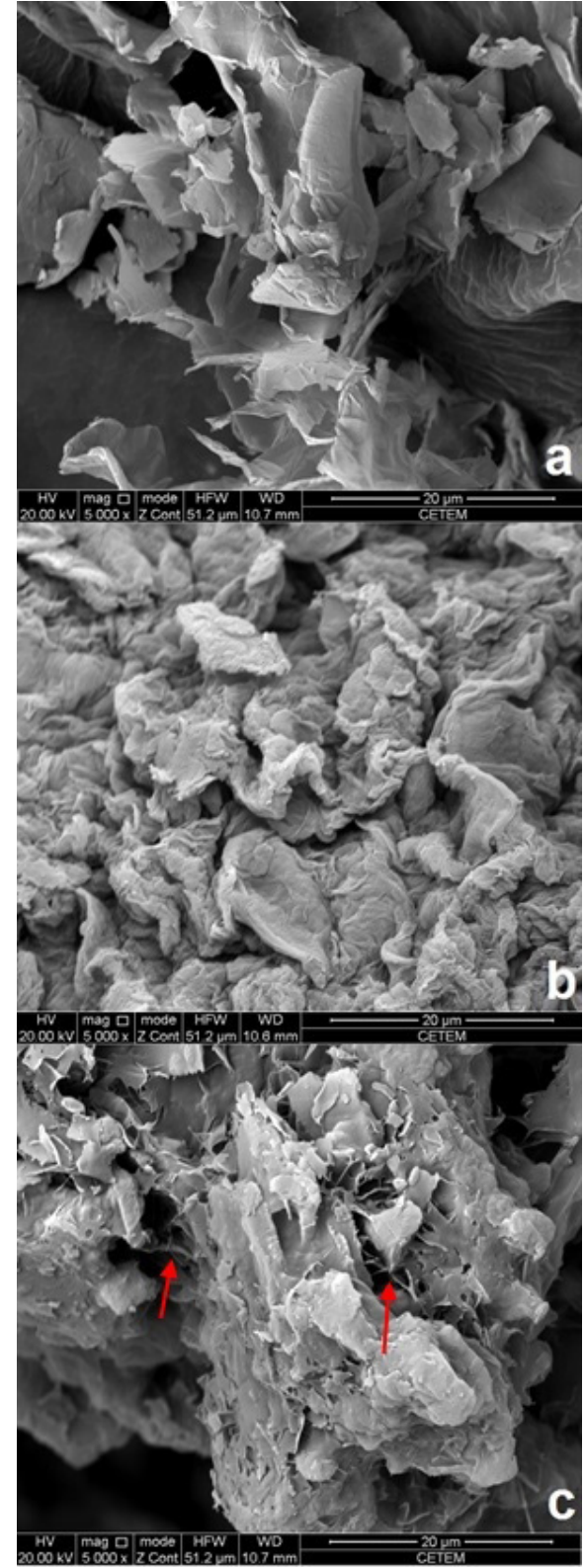

Figure 6. Images of SEM (a) graphite; (b) graphene oxide and (c) graphene modified with octadecylamine. 
octadecylamine. SEM images confirm the increase in distance between sheets already seen by XRD, when compared to packaged sheets of graphite. In addition, it is possible to observe the presence of a mass, which indicates the presence of a modifier on the surface of graphene sheets, which is in accordance with the results of TGA. This modification was designed to increase interfacial interaction with a polymer in a nanocomposite on a future study.

\section{Conclusions}

Surface modifications of different carbon nanostructures (multi-walled carbon nanotubes, graphene, and $\mathrm{C}_{60}$ fullerene) with octadecylamine were performed. The modified nanostructures were characterized in terms of morphology, chemical structure, molecular conformation and thermal properties. Routes of oxidation for each carbon nanostructure were successfully performed and verified by X-ray diffraction, FTIR and SEM measurements. Surface chemical modification of all carbon nanostructures with ODA were confirmed by XRD, FTIR, and SEM and demonstrated not to have altered the geometry of the nanoparticles. Thermogravimetric analysis revealed not only the success of surface oxidation of all carbon nanostructures, but showed us a very interesting trend too. Even if the reactivity order of the nanostructures is fullerene $>$ nanotubes $>$ graphene, the latter was the one that had the largest amount of ODA. That is the species of lower reactivity had the highest degree of modification, evidencing that the effect of specific surface area was greater than reactivity, and both oxidation and chemical modification method was very successful. In this way, we demonstrate that it is possible to perform chemical surface modification of carbon nanostructures without shape damage, making possible the maintenance of the properties inherent to each of the structures and allowing their use in polymer nanocomposites.

\section{Acknowledgements}

The authors thank Coordenação de Aperfeiçoamento de Pessoal de Nível Superior - Brazil (CAPES) - Finance Code 001, Conselho Nacional de Desenvolvimento Científico e Tecnológico (CNPq) for supporting of this work and CETEM - RJ for SEM images.

\section{References}

1. Niyogi S, Hamon MA, Hu H, Zhao B, Bhowmik P, Sen R, et al. Chemistry of single-walled carbon nanotubes. Acc Chem Res. 2002;35:1105-13.

2. Haddon RC. Comment on the relationship of the pyramidalization angle at a conjugated carbon atom to the $\sigma$ bond angles. J Phys Chem A. 2001;105:4164-5.

3. Taylor R, Walton DRM. The chemistry of fullerenes. Nature. 1993;363:685-93.

4. Tripathi SN, Rao GSS, Mathurb AB, Jasrab R. Polyolefin/graphene nanocomposites: a review. RSC Advances. 2017;7:23615-32.

5. Kausar A. Advances in polymer/fullerene nanocomposite: a review on essential features and applications. Polym Plast Technol Eng. 2017;56(6):594-605.

6. Imtiaz S, Siddiq M, Kausar A, Munthaa ST, Ambreenb J, Bibi I. A review featuring fabrication, properties and applications of Carbon Nanotubes (CNTs) reinforced polymer and epoxy nanocomposites. Chin J Polym Sci. 2018;36:445-61.

7. Sahoo NG, Rana S, Cho JW, Li L, Chan SH. Polymer nanocomposites based on functionalized carbon nanotubes. Prog Polym Sci. 2010;35:837-67.

8. Kroto HW, Heath JR, O'Brien SC, Curl RF, Smalley RE. $C_{60}$ : buckminsterfullerene. Nature. 1985;318:162-3.

9. Santos LJ, Rocha GP, Alves RB, Freitas RP. Fulereno $\left[\mathrm{C}_{60}\right]$ : química e aplicações. Quim Nova. 2010;3:680-93.

10. Jeong J, Kim WS, Park SI, Yoon TS, Chung BH. Synthesis and characterization of various-shaped $\mathrm{C}_{60}$ microcrystals using alcohols as antisolvents. J Phys Chem C. 2010;114:12976-81.

11. Cortes-Arriagada D, Sanhueza L, Bautista-HernandezA, SalazarVillanueva M, Anota EC. Chemical and physical viewpoints about the bonding in fullerene-graphene hybrid materials: interaction on pristine and Fe-Doped graphene. J Phys Chem C. 2019;123:24209-242019.

12. Iijima S. Helical microtubes of graphitic carbon. Nature. 1991;354:56-8.

13. Thostenson ET, Ren Z, Chou W. Advances in the science and technology of carbon nanotubes and their composites: a review. Compos Sci Technol. 2001;61:1899-912.

14. Moniruzzaman M, Winey KI. Polymer nanocomposites containing carbon nanotubes. Macromolecules. 2006;39:5194-205.

15. Ma PC, Siddiqui NA, Marom G, Kim JK. Dispersion and functionalization of carbon nanotubes for polymer-based nanocomposites: a review. Compos, Part A Appl Sci Manuf. 2010;41:1345-67.

16. Capek I. Dispersions, novel nanomaterial sensors and nanoconjugates based on carbon nanotubes. Adv Colloid Interface Sci. 2009;150:63-89.

17. Novoselov KS, Geim AK, Morozov SV, Jiang D, Zhang Y, Dubonos SV, et al. Electric field effect in atomically thin carbon films. Science. 2004;306:666-9.

18. Liu J, Tang J, Gooding JJ. Strategies for chemical modification of graphene and applications of chemically modified graphene. J Mater Chem. 2012;22:12435-52.

19. Xu M, Huang Q, Chen Q, Guo P, Sun Z. Synthesis and characterization of octadecylamine grafted multi-walled carbon nanotubes. Chem Phys Lett. 2003;375:598-604.

20. Mao Z, Wu W, Xie C, Zhang D, Jiang X. Lipophilic carbon nanotubes and their phase-separation in SBS. Polym Test. 2011;20:260-70.

21. Legramanti C, Silva EO, Tavares MIB. Crystallinity and molecular dynamics of EVA and multiwalled carbon nanotubes with different functionalizations. In: XI Brazilian MRS Meeting; 2012 Sep 23-27; Florianópolis, Brazil. Proceedings. Florianópolis: UFSC.

22. Yao H, Chu CC, Sue HJ, Nishimura R. Electrically conductive superhydrophobic octadecylamine-functionalized multiwall carbon nanotube films. Carbon. 2013;53:366-73.

23. Hummers WS, Offeman RE. Preparation of graphitic oxide. J Am Chem Soc. 1958;80:1339.

24. Marcano DC, Kosynkin DV, Berlin JM, Sinitskii A, Sun Z, Slesarev A, et al. Improved synthesis of graphene oxide. American Chemical Society Nanotation. 2010;4:4806-14.

25. Oh WC, Jung AR, Ko WB. The preparation and characterization of a silver-containing fullerenes $\mathrm{C}_{60}$. J Ceram Process Res. 2008;9:19-24.

26. Kim IH, Jeong YG. Polylactide/exfoliated graphite nanocomposites with enhanced thermal stability, mechanical modulus, and electrical conductivity. J Polym Sci, B, Polym Phys. 2010;48:850-8.

27. Zhang Y, Mark JE, Zhu Y, Ruoff RS, Scaefer DW. Mechanical properties of polybutadiene reinforced with octadecylamine modified graphene oxide. Polymer (Guildf). 2014;55:5389-95.

28. Gao Y, Chen X, Xu H, Zou Y, Gu R, Xu M, et al. Highly-efficient fabrication of nanoscrolls form functionalized graphene oxide by Langmuir_Blodgett method. Carbon. 2010;48:4475-82. 
29. Li W, Tang XZ, Zhang HB, Jiang ZG, Yu ZZ, Du XS, et al. Simultaneous surface functionalization and reduction of graphene oxide with octadecylamine for electrically conductive polystyrene composites. Carbon. 2011;49:4724-30.

30. Salam MA, Burk R. Synthesis and characterization of multiwalled carbon nanotubes modified with octadecylamine and polyethylene glycol. Arab J Chem. 2012;29:1-5.

31. Zhang SP, Song HO. Supramolecular graphene oxide-alkylamine hybrid materials: variation of dispersibility and improvement of thermal stability. New J Chem. 2012;36:1733-8.

32. Basiuk EV, Olmos OO, Torres FFC, Laguna VM, Zauco EA, Lee IP, et al. Green functionalization of pristine multi-walled carbono nanotubes with long-chain aliphatic amines. J Nanosci Nanotechnol. 2011;11:5546-54.

33. Liu YL, Su YH, Chang CM, Wang DM, Lai JY. Preparation and applications of Nafion-functionalized multiwalled carbon nanotubes for proton exchange membrane fuel cells. J Mater Chem. 2010;20:4409-16.

34. El-Khodary S, El-Enany GM, El-Okr M, Ibrahim M. Preparation and characterization of microwave reduced graphite oxide for high-performance supercapacitors. Electrochim Acta. 2014;150:269-78.

35. Chen Y, Tao J, Ezzeddine A, Mahfouz R, Al-Shahrani A, Alabedi G, Khashab NM. Superior performance nanocomposites from uniformly dispersed octadecylamine functionalized multi-walled carbon nanotubes. C- Journal of Carbon Research. 2015;1:58-76.

36. Wang Y, Zhang C, Du Z, Li H, Zou W. Synthesis of silver nanoparticles decorated MWCNTs and their application in antistatic polyetherimide matrix nanocomposite. Synth Met. 2013;182:49-55.

37. Sobhani Z, Behnam MA, Emami F, Dehghanian A, Jamhiri I. Photothermal therapy of melanoma tumor using multiwalled carbon nanotubes. Int J Med (Dubai). 2017;12:4509-17.

38. Peng X, Li Y, Zhang G, Zhang F, Fan X. Functionalization of graphene with nitrile groups by cycloaddition of tetracyanoethylene oxide. J Nanomater. 2013;1-5.

39. Rodbari RJ, Wendelbo R, Jamshidi LCLA, Hernández EP, Nascimento L. Study of physical and chemical characterization of nanocomposite polystyrene graphene oxide high acidity can be applied in thin films. J Chil Chem Soc. 2016;61(3):3120-4. 